\title{
Scanning Electron Microscopy of Murine Mammary Carcinoma Cells Treated with Anastrozole, Tamoxifen and the Combination
}

\author{
S.K. Majumdar*, J.M. Xanthopoulos* \\ *Department of Biology, Lafayette College, Easton, PA 18042, USA
}

Estrogen has a stimulatory effect on the growth of hormone-sensitive breast cancers. For the past twenty years, tamoxifen, an anitestrogen, has been the primary drug utilized in the treatment against estrogen receptor positive breast cancers [1]. However, tamoxifen has been shown to act as a partial estrogen agonist encouraging breast cancer growth, while other breast cancers develop resistance to tamoxifen due to the upregulation of estrogen receptors $[2,3]$. In response, research efforts have been focused on the development of aromatase inhibitors, like anastrozole, which work to lower the circulating levels of estrogen in post-menopausal women [3]. While some studies have been initiated to analyze the variable efficacies of anastrozole, tamoxifen, and the combination of the treatments, little is known about the cellular destruction caused by these agents [4]. By utilizing a murine mammary carcinoma cell line, namely 4T1, as a model of advanced stage breast cancer, the injurious effects of anastrozole, tamoxifen, and the combination treatments were evaluated.

Exponentially growing 4T1 cells were exposed to $50 \mu \mathrm{g} / \mathrm{ml}$ anastrozole, $3 \mu \mathrm{g} / \mathrm{ml}$ tamoxifen, and the combination of the same concentrations of both drugs for 24-48 hours. The cells were fixed with $2 \%$ glutaraldehyde, post-fixed in 1\% osmium tetroxide, dehydrated in an increasing ethanol series, critically dried, coated with gold-palladium and viewed under an ISI Super IIIA scanning electron microscope. Pictures were taken with Polaroid B-52 pan film and computer interface software.

When compared to controls (Fig. 1), anastrozole treated cells $(50 \mu \mathrm{g} / \mathrm{ml}, 48 \mathrm{hrs})$ exhibited various surface abnormalities, roundness and thinning lamellipodia (Fig. 3). At higher magnifications, 4T1 cells treated with anastrozole (Fig. 4), tamoxifen $(3 \mu \mathrm{g} / \mathrm{ml})$ (Fig. 5), and the combination treatment (Fig. 6) clearly demonstrate 4T1 surface ultrastructural alterations and damage when compared to control (Fig. 2). The drug treated cells demonstrated damage characteristic of apoptotic cells including blebs, apoptotic bodies, holes and cell lysing (Figs. 4,5,6). Additionally, both the combination and tamoxifen treatments showed a significant decrease in cell number when compared to control cells in a dosage and time dependent manner. Concurrent in vivo studies conducted in our laboratory have shown that mice inoculated with 4T1 cells treated with doses of tamoxifen demonstrated an increased life span when compared to non-drug treated mice, as well as fewer maladies [5]. Tamoxifen's observable adverse effects on cell proliferation may be due to its direct mechanism of estrogen reception inhibition [6].

References

[1] The Lancet, 359 (2002) 2131.

[2] J.F.R. Robertson, J. Steroid Biochem. \& Molec. Biol., 79 (2001) 209.

[3] P.E. Goss and K. Strasser, Drugs, 62 (2002) 957.

[4] Q. Lu et al., Breast Cancer Research and Treatment, 57 (1999) 183.

[5] J.M. Xanthopoulos et al., J BioMed Biotechnol, 2004, (in press).

[6] This work was supported by aid from Lafayette College. 

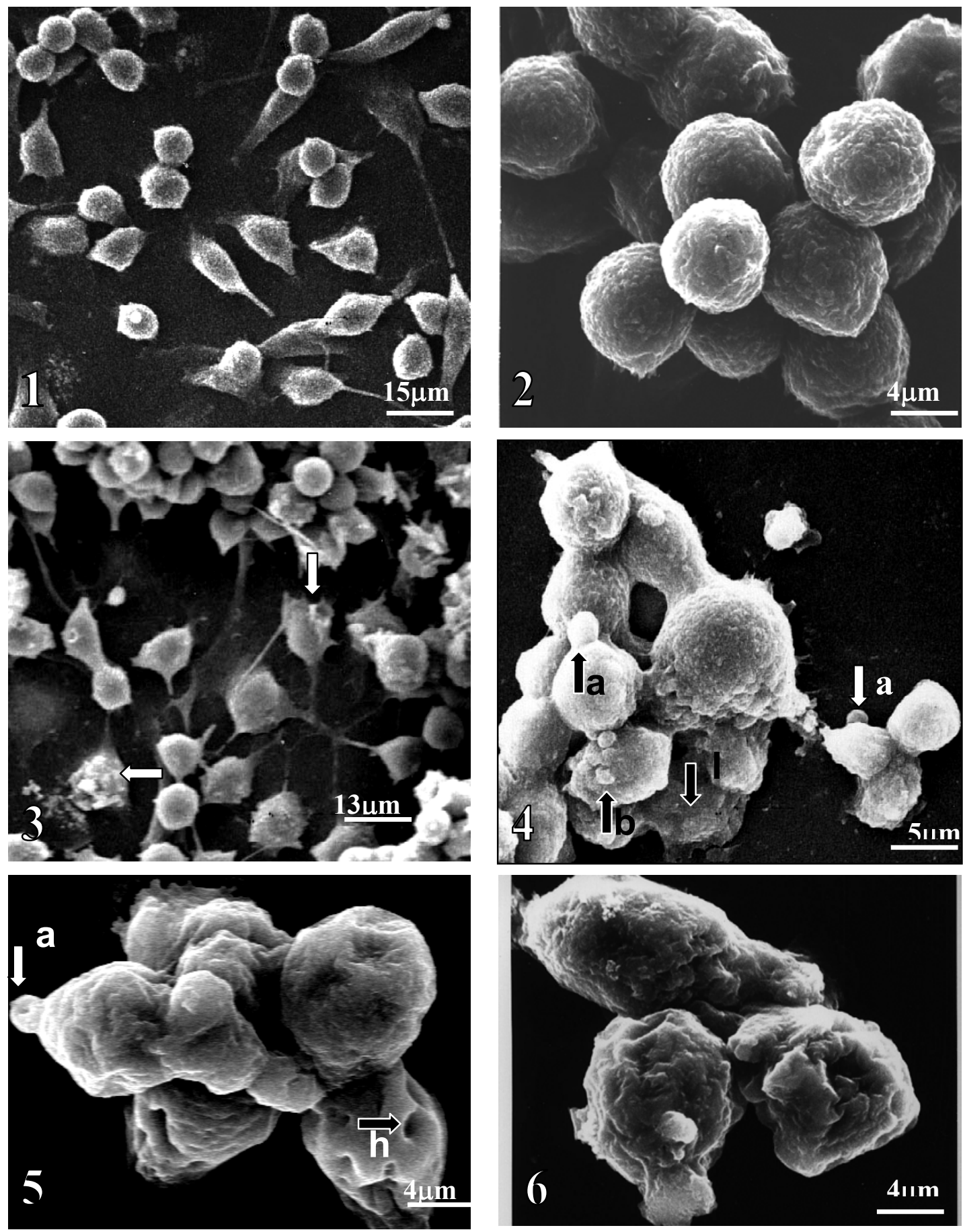

Figure 1: Characteristics of untreated $24 \mathrm{hrs}$ culture showing many rounded and flattened cells. Figure 2: Normal surface characteristics of untreated cells at $24 \mathrm{hrs}$.

Figure 3: Anastrozole $48 \mathrm{hrs}$ treated $(50 \mu \mathrm{g} / \mathrm{ml}) 4 \mathrm{~T} 1 \mathrm{cells}$ showing various surface abnormalities. Figure 4: Enlarged view of 48hrs treated anastrozole 4T1 cells depicting apoptotic bodies (a), blebs (b) and lysed cells (1).

Figure 5: Tamoxifen treated cells at $24 \mathrm{hrs}$ depicting irregularly shaped cells with apoptotic bodies (a) and holes (h).

Figure 6: Anastrozole and tamoxifen treated cells showing many surface ultrastructural changes. 